\title{
Corrected phase-type approximations for the workload of the MAP/G/1 queue with heavy-tailed service times
}

\author{
Eleni Vatamidou \\ EURANDOM and \\ Eindhoven University \\ of Technology \\ e.vatamidou@tue.nl
}

\author{
Ivo Adan \\ EURANDOM and \\ Eindhoven University \\ of Technology \\ i.j.b.f.adan@tue.nl
}

\author{
Maria Vlasiou \\ EURANDOM, CWI, and \\ Eindhoven University \\ of Technology \\ m.vlasiou@tue.nl
}

\author{
Bert Zwart \\ EURANDOM, CWI, \\ VU University Amsterdam, \\ Georgia Institute of \\ Technology, and Eindhoven \\ University of Technology \\ Bert.Zwart@cwi.nl
}

\begin{abstract}
In many applications, significant correlations between arrivals of load-generating events make the numerical evaluation of the load of a system a challenging problem. Here, we construct very accurate approximations of the workload distribution of the $\mathrm{MAP} / \mathrm{G} / 1$ queue that capture the tail behavior of the exact workload distribution and provide a small relative error. Motivated by statistical analysis, we assume that the service times are a mixture of a phase-type and a heavy-tailed distribution. With the aid of perturbation analysis, we derive our approximations as a sum of the workload distribution of the MAP/PH/1 queue and a heavytailed component that depends on the perturbation parameter. We refer to our approximations as corrected phase-type approximations, and we exhibit their performance with a numerical study.
\end{abstract}

Keywords: Markovian Arrival Process (MAP); Workload distribution; Heavy-tailed service times; Tail asymptotics; Perturbation analysis.

\section{INTRODUCTION}

The evaluation of the workload of a MAP/G/1 queue is an important problem that has been widely studied in the literature. For an extensive review see [9]. Although closedform expressions for the evaluation of the workload are available, they are practical only in the case of phase-type (PH) service times. When the workload distribution cannot be computed exactly, it needs to be approximated. Here, we develop a new method to construct reliable approximations for the workload distribution for heavy-tailed service times.

Two main directions for approximating the workload distribution are $\mathrm{PH}$ and asymptotic approximations. When the service times are light-tailed, a common approach to approximate the workload with high accuracy is by approximating the service time distribution with a $\mathrm{PH}$ one 8, 13. We refer to these methods as phase-type approximations, because the approximate workload distribution has a $\mathrm{PH}$ representation [10]. However, in many cases, a heavy-tailed distribution is most appropriate to model the service times [6, 12]. For the special class of subexponential service times, asymptotic approximations for the workload distribution are available, which provide a good fit only at the tail [3, 7.
In this paper, we develop approximations of the workload distribution for heavy-tailed service times that maintain the computational tractability of $\mathrm{PH}$ approximations, capture the correct tail behavior and provide small absolute and relative errors. Also, they have the advantage that finite higher-order moments for the service times are not required. In order to achieve these desirable characteristics, our key idea is to use a mixture model for the service times.

The idea of our approach stems from fitting procedures of the service time distribution to data. Heavy-tailed statistical analysis suggests that only a small fraction of the upper-order statistics of a sample is relevant for estimating tail probabilities [11. The remaining data set may be used to fit the bulk of the distribution. Since PH distributions are dense in the class of all positive definite probability distributions 4, a natural choice is to fit a $\mathrm{PH}$ distribution to the remaining data set [5. As a result, a mixture model for the service times is a natural assumption.

In short, we consider the service time distribution as a mixture of a $\mathrm{PH}$ distribution and a heavy-tailed one. As "base" model we use the model appearing when all heavytailed customers are removed, and we interpret the heavytailed term of the mixture model as perturbation of the $\mathrm{PH}$ one. Using perturbation analysis, we find our approximations for the workload in the mixture model as a sum of the workload of the base model and a heavy-tailed component that depends on the perturbation parameter. In a previous study (cf. [14]), we carried out this project for Poisson arrivals. Here we develop an extension to MAP's.

The rest of the paper is structured as follows. In Section 2. we introduce the notation for the model under consideration, and in Section 3 we present the algorithm to construct our approximations. In Section 4 we give their formulas and we also specialize to the M/G/1 queue. Finally, in Section 5 . we perform an illustrative numerical experiment.

\section{MODEL DESCRIPTION}

We consider a single server queue with FIFO discipline, where customers arrive according to a Markovian Arrival Process (MAP) with $N$ states. The regulating Markov chain $\left\{Z_{n}\right\}_{n \geq 0}$ has an irreducible transition probability matrix $\mathbf{P}$ and stationary distribution $\boldsymbol{\pi}$. Transitions from state $i$ occur at an exponential rate $\lambda_{i}$ (directed to state $j$ with probability $p_{i j}$ ). With probability $q_{i}$, a transition from $i$ corresponds to an arrival of a (real) customer with service time distribution $F(t)$ (independent of the state), and otherwise, it 
corresponds to an arrival of a (dummy) customer with zero service time. So the service time distribution of a customer arriving in state $i$ is $G_{i}(t)=q_{i} F(t)+1-q_{i}$. Observe that if the service time distribution of a real customers $F(t)$ was depending on the state of the system then we would allow for cross-correlations between the arrivals and the services, which is not the case in our model.

In matrix form, the above quantities can be written as $\mathbf{Q}=\operatorname{diag}\left(q_{1}, \ldots, q_{N}\right), \boldsymbol{\Lambda}=\operatorname{diag}\left(\lambda_{1}, \ldots, \lambda_{N}\right)$ and $\widetilde{\mathbf{G}}(s)=$ $\widetilde{F}(s) \mathbf{Q}+(\mathbf{I}-\mathbf{Q})$, where $\widetilde{F}(s)$ denotes the Laplace-Stieltjes transform (LST) of the service time distribution $F(t)$ of a real customer, and $\mathbf{I}$ stands for the identity matrix with dimension $N$. Finally, let $\mu$ be the mean of the service time distribution $F(t)$. We assume that the system is stable, namely $\boldsymbol{\pi}\left(\boldsymbol{\Lambda}^{-1}-\mu \mathbf{Q}\right) \mathbf{e}>0$, where $\mathbf{e}$ is the column vector with all elements equal to 1 . If $\widetilde{\phi}_{i}(s)$ denotes the LST of the steady-state workload in state $i$, the following theorem holds for the transform vector $\widetilde{\boldsymbol{\Phi}}(s)=\left[\widetilde{\phi}_{1}(s), \ldots, \widetilde{\phi}_{N}(s)\right]$ (cf. Th. 3.1 in 2]).

THEOREM 2.1. If the system is stable, there exists a unique vector $\mathbf{u}=\left[u_{1}, \ldots, u_{N}\right]$, such that $\widetilde{\boldsymbol{\Phi}}(s)$ satisfies

$$
\begin{aligned}
\widetilde{\boldsymbol{\Phi}}(s)(\widetilde{\mathbf{G}}(s) \mathbf{P} \boldsymbol{\Lambda}+s \mathbf{I}-\boldsymbol{\Lambda}) & =s \mathbf{u}, \\
\widetilde{\boldsymbol{\Phi}}(0) \mathbf{e} & =1 .
\end{aligned}
$$

To determine the unknown vector $\mathbf{u}$ we have (cf. Th. 3.2 \& 3.3. in [2]):

\section{THEOREM 2.2. It holds that}

1. $\operatorname{det}(\widetilde{\mathbf{G}}(s) \mathbf{P} \boldsymbol{\Lambda}+s \mathbf{I}-\boldsymbol{\Lambda})=0$ has exactly $N$ roots $s_{i}$, with $s_{1}=0$ and $\operatorname{Re}\left(s_{i}\right)>0, i=2, \ldots, N$.

2. Let $\mathbf{a}_{i}$ be non-zero column vectors satisfying

$$
\left(\widetilde{\mathbf{G}}\left(s_{i}\right) \mathbf{P} \boldsymbol{\Lambda}+s_{i} \mathbf{I}-\boldsymbol{\Lambda}\right) \mathbf{a}_{i}=0, \quad i=2, \ldots, N .
$$

Then, provided all $s_{i}$ are distinct, $\mathbf{u}$ is the unique solution to the $N$ linear equations:

$$
\begin{aligned}
\mathbf{u} \boldsymbol{\Lambda}^{-1} \mathbf{e} & =\boldsymbol{\pi}\left(\boldsymbol{\Lambda}^{-1}-\mu \mathbf{Q}\right) \mathbf{e}, \\
\mathbf{u a}_{i} & =0, \quad i=2, \ldots, N .
\end{aligned}
$$

Combining the results of Theorems 2.1 and 2.2 the LST of the total workload $V$ in the system is

$$
\widetilde{v}(s)=\frac{s \cdot \mathbf{u} \cdot \operatorname{adj}(\widetilde{\mathbf{G}}(s) \mathbf{P} \boldsymbol{\Lambda}+s \mathbf{I}-\boldsymbol{\Lambda}) \mathbf{e}}{\operatorname{det}(\widetilde{\mathbf{G}}(s) \mathbf{P} \boldsymbol{\Lambda}+s \mathbf{I}-\boldsymbol{\Lambda})},
$$

where adj denotes the adjoint of a square matrix.

We now assume that the service time distribution of a real customer has the form $F(t)=(1-\epsilon) F_{p}(t)+\epsilon F_{h}(t), \epsilon \in[0,1)$, for some $\mathrm{PH}\left(F_{p}(t)\right)$ and heavy-tailed $\left(F_{h}(t)\right)$ distributions, with finite means $\mu_{p}$ and $\mu_{h}$, respectively. Theorem 2.2 guarantees that the RHS of (1) in this model with mixed service time distribution is well-defined in the positive halfplane. However, if the LST $\widetilde{F}_{h}(s)$ does not have a closedform expression (e.g.Pareto), Laplace inversion of (1) cannot be applied to find the distribution of the workload $V_{\epsilon}$ in this mixture model.

In the next section, we describe how to create an approximation for $V_{\epsilon}$, by approximating its LST.

\section{APPROACH}

The steps to construct our approximations are:

1. Use a PH approximation as base model.

(a) Set $\epsilon=0$ and $\widetilde{F}_{p}(s)=q_{n}(s) / p_{m}(s)$, where $q_{n}(s)$ and $p_{m}(s)$ are polynomials of degrees $m$ and $n$ respectively, with $n \leq m-1$, so that $\widetilde{F}_{p}(s)$ is the LST of a PH-distribution.

(b) Use Theorem 2.2 to determine the vector $\mathbf{u}$ and find the adjoint matrix $\operatorname{adj}(\widetilde{\mathbf{G}}(s) \mathbf{P} \boldsymbol{\Lambda}+s \mathbf{I}-\boldsymbol{\Lambda})$.

(c) Find the LST $\widetilde{v}(s)$ (see (1)) as

$$
\widetilde{v}(s)=\frac{\mathbf{u e} \prod_{j=1}^{m r}\left(s+y_{j}\right)}{\prod_{j=1}^{m r}\left(s+x_{j}\right)},
$$

where $\operatorname{Re}\left(y_{j}\right)>0, \operatorname{Re}\left(x_{j}\right)>0, j=1, \ldots, m r$, and $r$ is an non-negative integer smaller than or equal to the rank of $\mathbf{P}$.

(d) Apply Laplace inversion to (2) to find analytically $\mathbb{P}(V>t)$.

2. Find the parameters of the mixture model as perturbation of the base model's ones (parameters affected by the perturbation bear an index $\epsilon$ ).

(a) For the matrix $\widetilde{\mathbf{G}}_{\epsilon}(s) \mathbf{P} \boldsymbol{\Lambda}+s \mathbf{I}-\boldsymbol{\Lambda}$, find its determinant and its adjoint matrix .

(b) Evaluate the vector $\mathbf{u}_{\epsilon}$ and the roots $s_{\epsilon, i}, i=$ $1, \ldots, N$, using an extension of Theorem 2.2 (omitted due to space limitations).

3. Find the LST of the workload $V_{\epsilon}$ as perturbation of $\widetilde{v}(s)$, by keeping only up to $\epsilon$-order terms, i.e.,

$$
\widetilde{v}_{\epsilon}(s)=\widetilde{v}(s)+\epsilon \widetilde{v}(s) k(s)+O\left(\epsilon^{2}\right),
$$

where $k(s)$ is well-defined for positive values.

Our proposed approximations are constructed by applying Laplace inversion to the up to $\epsilon$-order terms of (3). In the next section, we give their formulas.

\section{CORRECTED PH APPROXIMATIONS}

Let $B^{e}$ and $C^{e}$ be the generic stationary excess $\mathrm{PH}$ and heavy-tailed service times, respectively. Moreover, let $E_{\lambda}$ be an exponential r.v. with rate $\lambda$, and let $V^{\prime}$ be independent and follow the same distribution of $V$. The next theorem shows that each term in the Laplace inverse $\mathcal{L}^{-1}\{\widetilde{v}(s) k(s)\}$ has a probabilistic interpretation.

THEOREM 4.1. There exist unique coefficients $\alpha, \beta, \gamma$, $\alpha_{j}, \beta_{j}, \gamma_{j}, j=1, \ldots, m r$ and $\delta_{i}, \eta_{i}, \theta_{i}, i=2, \ldots, N$, s.t.

$$
\begin{aligned}
& \mathcal{L}^{-1}\{\widetilde{v}(s) k(s)\}=\frac{1}{\mathbf{u e}}\left(\beta\left(\mu_{p} \mathbb{P}\left(V+B^{e}>t\right)-\mu_{h} \mathbb{P}\left(V+C^{e}>t\right)\right)\right. \\
+ & \sum_{j=1}^{m r} \beta_{j}\left(\mu_{p} \mathbb{P}\left(V+B^{e}+E_{y_{j}}>t\right)-\mu_{h} \mathbb{P}\left(V+C^{e}+E_{y_{j}}>t\right)\right) \\
+ & \sum_{i=2}^{N} \eta_{i}\left(\mu_{p} \mathbb{P}\left(t<V+B^{e}<t+E_{s_{i}}\right)-\mu_{h} \mathbb{P}\left(t<V+C^{e}<t+E_{s_{i}}\right)\right) \\
+ & \gamma\left(\mu_{p} \mathbb{P}\left(V+V^{\prime}+B^{e}>t\right)-\mu_{h} \mathbb{P}\left(V+V^{\prime}+C^{e}>t\right)\right)
\end{aligned}
$$




$$
\begin{aligned}
& +\sum_{j=1}^{m r} \gamma_{j}\left(\mu_{p} \mathbb{P}\left(V+V^{\prime}+B^{e}+E_{y_{j}}>t\right)-\mu_{h} \mathbb{P}\left(V+V^{\prime}+C^{e}+E_{y_{j}}>t\right)\right) \\
& +\sum_{i=2}^{N} \theta_{i}\left(\mu_{p} \mathbb{P}\left(t<V+V^{\prime}+B^{e}<t+E_{s_{i}}\right)\right. \\
& \left.\quad-\mu_{h} \mathbb{P}\left(t<V+V^{\prime}+C^{e}<t+E_{s_{i}}\right)\right)+\alpha \mathbb{P}(V>t) \\
& \left.\quad+\sum_{j=1}^{m r} \alpha_{j} \mathbb{P}\left(V+E_{y_{j}}>t\right)+\sum_{i=2}^{N} \delta_{i} \mathbb{P}\left(t<V<t+E_{s_{i}}\right)\right) .
\end{aligned}
$$

Remark 1. In Theorem 4.1, we assumed for convenience that all $y_{j}$ are simple, but the result can be generalized to roots with multiplicity greater than one. Also, the unique coefficients are found in a straightforward way, but we omit the details.

Remark 2. We assumed that all $s_{i}$ and $y_{j}$ are real-valued. If e.g. $s_{2}$ is complex, then we write $E_{R e\left(s_{2}\right)}$ instead of $E_{s_{2}}$. The imaginary part cancels out when we combine each complex root with its conjugate.

Let $\hat{V}_{\epsilon}$ denote the approximation of $V_{\epsilon}$. Following the previous result, we have

Definition 1. The corrected PH approximation is

$$
\mathbb{P}\left(\hat{V}_{\epsilon}>t\right):=\mathbb{P}(V>t)+\epsilon \mathcal{L}^{-1}\{\widetilde{v}(s) k(s)\},
$$

where $\mathcal{L}^{-1}\{\widetilde{v}(s) k(s)\}$ is given by Theorem 4.1 and $\mathbb{P}(V>t)$ follows from step (1d).

For the M/G/1, the coefficients of Theorem 4.1 are directly obtained from the parameters of the system.

Corollary 1. In case of Poisson arrivals with rate $\lambda$, the corrected PH approximation takes the form

$$
\begin{aligned}
& \mathbb{P}\left(\hat{V}_{\epsilon}>t\right):=\mathbb{P}(V>t)+\epsilon \frac{\lambda}{1-\lambda \mu_{p}}\left(\left(\mu_{p}-\mu_{h}\right) \mathbb{P}(V>t)\right. \\
& \left.\quad+\mu_{h} \mathbb{P}\left(V+V^{\prime}+C^{e}>t\right)-\mu_{p} \mathbb{P}\left(V+V^{\prime}+B^{e}>t\right)\right) .
\end{aligned}
$$

Using a test model, in Section 5 we check the performance of our approximations.

\section{NUMERICAL EXAMPLE}

We consider a MAP with Erlang-2 distributed interarrival times, where the exponential phases have both rate $\lambda$, namely $\lambda_{i}=\lambda, i=1,2$. For the service times, we use a mixture of an $\operatorname{Exp}(\nu)$ distribution and a heavy-tailed one (cf. [1]) with $\operatorname{LST} \widetilde{F}_{h}(s)=1-\frac{s}{(\kappa+\sqrt{s})(1+\sqrt{s})}$. The exact workload distribution for this mixture of distributions can be calculated by following a similar idea as the proof of Th. 9 in [14]. For our numerical examples, we select $\kappa=2, \nu=3$ and $\epsilon=0.01$.

From Table 1 we observe that the corrected $\mathrm{PH}$ approximation yields a significant improvement to its $\mathrm{PH}$ counterpart. The difference between the exact tail probabilities of the workload and the corrected $\mathrm{PH}$ approximation is $O\left(10^{-4}\right)$, while for the $\mathrm{PH}$ approximation it is $O\left(10^{-2}\right)$, for all values. The magnitude of the improvement we achieve with the corrected $\mathrm{PH}$ is evident by looking at the relative errors of the involved approximations. The relative error of the $\mathrm{PH}$ easily reaches values close to 1 , while the corrected $\mathrm{PH}$ gives a relative error $O(\epsilon)$.

\begin{tabular}{|r|clc|}
\hline$t$ & exact & PH & corrected-PH \\
\hline 0 & 0.837500 & 0.833333 & 0.837213 \\
5 & 0.061452 & 0.031781 & 0.060882 \\
10 & 0.023269 & 0.001212 & 0.023544 \\
15 & 0.017579 & 0.000046 & 0.017862 \\
20 & 0.014979 & $1.76 \times 10^{-6}$ & 0.014091 \\
25 & 0.013301 & $6.72 \times 10^{-8}$ & 0.013126 \\
30 & 0.012090 & $2.56 \times 10^{-9}$ & 0.011867 \\
35 & 0.011162 & $9.78 \times 10^{-11}$ & 0.010943 \\
40 & 0.010419 & $3.73 \times 10^{-12}$ & 0.010220 \\
45 & 0.009809 & $1.42 \times 10^{-13}$ & 0.009601 \\
50 & 0.009294 & $5.42 \times 10^{-15}$ & 0.009106 \\
\hline
\end{tabular}

Table 1: Tail probabilities of the exact workload, the PH and the corrected $\mathbf{P H}$ approximations for $\epsilon=0.01$ and load 0.8375 .

\section{REFERENCES}

[1] Abate, J. And Whitt, W. (1999). Explicit $M / G / 1$ waiting-time distributions for a class of long-tail service-time distributions. Oper.Res.Lett. 25, 25-31.

[2] Adan, I.J.B.F. And Kulkarni, V.G. (2003). Single-server queue with Markov-dependent inter-arrival and service times. QUESTA 45, 113-134.

[3] Asmussen, S. (2000). Ruin Probabilities

[4] Asmussen, S. (2003). Applied Probability and Queues. Springer-Verlag, New York.

[5] Asmussen, S., Nerman, O. And Olson, M. (1996). Fitting phase-type distributions via the EM algorithm. Scand. J. Stat. 23, 419-441.

[6] Embrechts, P., Klüppelberg, C. And Mikosch, T. (1997). Modelling Extremal Events Springer-Verlag, Berlin.

[7] Embrechts, P. And Veraverbeke, N. (1982). Estimates for the probability of ruin with special emphasis on the possibility of large claims. Insur. Math. Econ. 1, 55-72.

[8] Feldmann, A. And Whitt, W. (1998). Fitting mixtures of exponentials to long-tail distributions to analyze network performance models. Perform. Evaluation. 31, 245-279.

[9] Lucantoni, D. M. (1993). The BMAP/G/1 queue: A tutorial.

[10] Ramaswami, V. (1990). From the matrix-geometric to the matrix-exponential. QUESTA 6, 229-260.

[11] Resnick, S. I. (2007). Heavy-Tail Phenomena: Probabilistic and Statistical Modeling. Springer, NY.

[12] Rolski, T., Schmidli, H., Schmidt, V. And Teugels, J. (1999). Stochastic Processes for Insurance and Finance. John Wiley \& Sons Ltd.

[13] Starobinski, D. And Sidi, M. (2000). Modeling and analysis of power-tail distributions via classical teletraffic methods. QUESTA 36, 243-267.

[14] Vatamidou, E., Adan, I., Vlasiou, M. And Zwart, B. (2013). Corrected phase-type approximations of heavy-tailed risk models using perturbation analysis. Insur Math Econ 53, 366-378. 